\title{
P04-1-82 Poster session
}

\section{Selective elimination of slow motor neurons in mice progressively induces a kinetic tremor that resembles patients with essential tremor}

\section{Lisa Ohgaki ${ }^{1}$, Daijiro Inomata ${ }^{1}$, Tenkei Koyama ${ }^{1}$, Tomoyuki Yamanaka ${ }^{2}$, Shigeyoshi Itohara ${ }^{3}$, Hidemi Misawa ${ }^{1}$}

${ }^{I}$ Division of Pharmacology, Faculty of Pharmacy, Keio University, Japan, ${ }^{2}$ Laboratory of Structural Neuropathology, Graduate School of Brain Science, Doshisha University, Japan, ${ }^{3}$ Laboratory for Behavioral Genetics, RIKEN Brain Science Institute, Japan

VAChT-Cre is a Cre-driver mouse line that can direct DNA recombination in postnatal FR-type (fast-twitch fatigue resistant) or S-type (slow-twitch fatigue resistant) motor neurons (hereinafter referred to as "slow motor neurons ") innervating type-I or -IIa muscle fibers (Fig. 1; Misawa et al., genesis, 54, 568-572, 2016). In order to express cytotoxic substances selectively in slow motor neurons, VAChT-Cre mice were crossbred with NSE-DTA mice in which diphtheria toxin A (DTA) was expressed after Cre-mediated loxP-site excision. The resultant VAChT-Cre;NSE- DTA mice were born normally and grow healthy, but started voluntary shaking around 20 weeks of age. Reduced body weight and hunched back posture in the spine (kyphosis) were observed around this age. Muscular atrophy was evident in " red muscle " including the soleus muscle, however " white muscle" such as the extensor digitorum longus muscle was normal. The tremor was kinetic in nature and became progressively worsen with ageing. The tremor was most conspicuous in head and neck.

Tremor frequencies and amplitudes were analyzed using an accelerometer (glued to hanging box) followed by fast Fourier transformation (FFT). The power spectrum analysis revealed that the tremor amplitude peaked at 18- to 22- $\mathrm{Hz}$, which was different from tremor frequency induced by a typical tremor-inducing drug harmaline (peaked at 11- to 15- $\mathrm{Hz}$ ), implying different causes for the tremor oscillation. The observed tremor phenotype in VAChT-Cre;NSE-DTA mice is reminiscent of essential tremor (ET) in humans: the most frequent movement disorders affecting elderly people with unknown cause. Possible mechanisms and sites of tremor origin in VAChT-Cre;NSE-DTA mice and it's relation to ET will be discussed. 